\title{
Predictive Validity of the Children's Attributional Styles Questionnaire: Linkages with Reactions to an In Vivo Peer Evaluation Manipulation
}

\author{
Albert Reijntjes · Maja Dekovic • \\ Marjolijn Vermande $\cdot$ Michael J. Telch
}

Published online: 15 February 2007

(C) Springer Science+Business Media, LLC 2007

\begin{abstract}
The present study examined the predictive validity of the Children's Attributional Styles Questionnaire (CASQ) by investigating associations between children's attributional style and their cognitive appraisals of an in vivo peer evaluation manipulation. Participants $(n=92)$, ages 10-13, played a computer contest based on the television show Survivor and were randomized to either a peer rejection (i.e., receiving the lowest total 'likeability' score from a group of peer-evaluators), a peer success (i.e., receiving the highest score), or a neutral peer feedback condition. Children reporting a more depressogenic attributional style displayed more negative cognitive appraisals of failure feedback. However, no linkage was found between children's enhancing attributional style and their appraisal of success feedback. These findings provide partial support for the predictive validity of the CASQ.
\end{abstract}

Keywords Children's Attributional Styles Questionnaire ·

Pre-adolescent children · Predictive validity · Depressive symptoms .

Peer rejection · Survivor contest

\footnotetext{
A. Reijntjes · M. Dekovic $\cdot$ M. Vermande

Department of Psychological Development in Context, Utrecht University, Heidelberglaan, Utrecht, The Netherlands
}
A. Reijntjes
e-mail: a.h.a.reijntjes@fss.uu.nl
M. Dekovic
e-mail: m.dekovic@fss.uu.nl
M. Vermande
e-mail: m.vermande@fss.uu.nl

M. J. Telch ( $\square)$

Department of Psychology, University of Texas at Austin, 1 University Station, Mail Code A8000,

Austin, TX 78712, USA

e-mail: telch@austin.utexas.edu 
Cognitive diathesis-stress models of depression (e.g., Abramson, Metalsky, \& Alloy, 1989; Beck, 1967; Monroe \& Simons, 1991) assert that people with certain negative cognitive styles are particularly susceptible to the depressogenic effects of life stress. For instance, Beck (1967) postulated that negative self-schema containing cognitive distortions or dysfunctional attitudes (e.g., 'I am nothing if a person I love doesn't love me') represents a cognitive diathesis which, in the presence of negative life events, constitutes a vulnerability to the onset of depression.

In the reformulated version of learned helplessness theory (Abramson, Seligman, \& Teasdale, 1978), a person's characteristic way of explaining negative events was elevated to a central position in explaining susceptibility to depression. Specifically, the tendency to make internal, stable, and global attributions for negative life events was proposed as a risk factor for the development of depression. In the more recently advanced hopelessness theory of depression (Abramson et al., 1989), the internal attributional dimension was demoted to a contributory cause of one particular symptom of depression (i.e., low self-esteem); whereas the global-specific and the stable-unstable dimensions by themselves were posited to be related to the risk for development of hopelessness and depressive symptoms. In line with this formulation, Joiner and Rudd (1996) showed that the attributional dimensions of stability and globality reflect the same underlying factor, but internality does not.

A stable attribution explains the cause of a negative event in terms of long-lasting, constant factors ('it will always be like this'), whereas an unstable attribution is transient ('it was only this one time, it will not happen again'). A global attribution explains the cause of a negative event in terms that affect a wide range of situations ('it will have a bearing on everything I do'), whereas a specific attribution is more circumscribed ('I failed on that test because I was very tired that day'). The stability and globality dimensions combine to form a "generality" composite dimension. The tendency to attribute bad events to stable and global causes is coined depressogenic attributional style.

Analogous to the hopelessness theory of depression onset, Needles and Abramson (1990) advanced a model of a recovery process from depression which asserts that the occurrence of positive life events may interact with 'enhancing attributional style' to offset hopelessness, thereby facilitating recovery from depression. Enhancing attributional style is defined as the tendency to make stable and global attributions for positive, rather than negative, life events. Importantly, research has provided evidence to suggest that depressogenic and enhancing attributional styles are separate constructs, with attributional style for positive events being more weakly associated with onset of depression than attributional style for negative events (see Sweeney, Anderson, \& Bailey (1986), for a meta-analysis of 104 studies).

Among children, studies investigating the hopelessness theory of depression have received mixed support. Several investigators have found that the interaction of Negative Attributional Style by Negative Life Events predicted subsequent increases in depression (e.g., Dixon \& Ahrens, 1992; Panak \& Garber, 1992); whereas negative findings have been reported by other investigators (e.g., Cole \& Turner, 1993; Hammen, Adrian, \& Hirito, 1988). Two long-term longitudinal studies have yielded both supportive and unsupportive results over time within the same study (Nolen-Hoeksema, Girgus, \& Seligman, 1986, 1992).

The few studies that have directly tested Needles and Abramson's (1990) recovery model of depression (e.g., Edelman, Ahrens, \& Haaga, 1994; Johnson, Crofton, \& Feinstein, 1996; Johnson, Han, Douglas, Johannet, \& Russell, 1998) have also yielded inconclusive results. For instance, while the interaction between enhancing attributional 
style and the occurrence of positive life events predicted recovery from depressive symptoms in the Edelman et al. (1994) study, this relationship was not mediated by hopelessness. Moreover, Johnson and co-workers (1996) observed that the combined main effects of an enhancing attributional style and the presence of positive events, but not their interaction, predicted increases in hopefulness and subsequent decreases in depressive symptoms. Finally, in the one study using a sample of children and adolescents, Voeltz and colleagues (Voeltz, Haeffel, Joiner, \& Wagner, 2003) observed that changes in hopelessness were predicted by the interaction between a depressiogenic attributional style for negative events and an enhancing attributional style for positive events.

During the past two decades, the Children's Attributional Styles Questionnaire (CASQ; Seligman et al., 1984) has been the most widely used instrument to measure attributional styles in children and adolescents, ages 7 through 17 (see Joiner \& Wagner (1995), for a review). The CASQ is a 48-item self-report questionnaire designed to assess causal attributions for hypothetical positive (24 items) and negative events (24 items). Each item depicts a hypothetical situation (e.g., "you get a bad grade in school") followed by two statements indicating why the event may have happened (e.g., "I am not a good student", or "teachers give hard tests"). Participants choose the response that best explains why the event might have occurred. Sixteen questions pertain to each of the three attributional dimensions of internality, stability, and globality.

Several studies have investigated the psychometric properties of the CASQ (e.g., Gotlib, Lewinsohn, Seeley, Rohde, \& Redner, 1993; Nolen-Hoeksema et al., 1986, 1992; Panak \& Garber, 1992; Seligman et al., 1984). Modest internal consistencies have been observed for the negative (.47 to .67) and positive (.45 to .73) composite scores. Testretest reliabilities over a 1-year period for these two composite scores were .48 and .54 , respectively (Gotlib et al., 1993).

The CASQ has demonstrated good concurrent criterion-related validity. Specifically, a meta-analytic study involving 7500 subjects showed that children and adolescents higher in depression, relative to their peers, displayed more internal, stable, and global attributions for failure, and more external, unstable, and specific attributions for success (Gladstone \& Kaslow, 1995). Effect sizes for these associations ranged from moderate to large (Cohen, 1977).

To our knowledge, the predictive validity of the CASQ has yet to be examined. To the extent that depressogenic and enhancing attributional style are dispositional propensities to interpret (the causes of) positive and negative life events in a certain fashion, adequate predictive validity presupposes at least a moderate degree of correspondence between the trait measure and the child's cognitive appraisal of an actually experienced salient event in real time. The present study was designed to provide information on the predictive validity of the CASQ in a community sample of pre-adolescent children, by examining the linkage between children's scores on the CASQ and their cognitive appraisals in response to an experimentally manipulated peer evaluation outcome.

Toward this aim, we devised a peer feedback manipulation based on the television show Survivor. Peer evaluation was selected as the domain of inquiry because peer praise and peer rejection likely rank as salient and personally relevant (i.e., egoinvolving) events. Moreover, peer evaluation has a high degree of ecological validity, in that rejection and praise by peers are common emotion-eliciting events in childhood (Coie, 1990). We selected children between the ages of 10-13 because peers assume great salience in this age group, as evidenced by data indicating that by age 11 nearly $50 \%$ of children's social activities involve peers (Grusec \& Lytton, 1988). 
In brief, participants were led to believe that they would participate in an internet version of the television show Survivor and would compete with four same-sex players of comparable age from four different schools in the same area (all of which were computerized fictitious co-players). They were informed that all participants would be evaluated by a team of 16 same-age peer evaluators consisting of eight boys and eight girls. Specifically, each evaluator would give them a score between 0 and 100, with higher scores reflecting higher levels of perceived likeability. Participants were randomized to one of the following three peer evaluation feedback conditions: (a) success feedback (i.e., having obtained the highest total score), (b) failure feedback (i.e., having obtained the lowest total score), or (c) neutral feedback (i.e., having obtained neither the highest nor the lowest score).

In sum, the present study examined the predictive validity of children's attributional style for positive and negative events, as assessed by the CASQ. In so doing, we also examined if changes in state mood in response to the positive/negative feedback manipulation were associated with the child's attributional style for positive/negative events and/or actual attributions made for the positive/negative Survivor evaluation feedback. Moreover, given their likely role in affecting children's cognitive responses to the peer feedback outcome, the role of children's level of depressive symptoms and their peer-nominated social standing in the peer group were also examined. Finally, we examined the potential qualifying effects of gender and age.

We expected that children's depressogenic, but not enhancing, attributional style would predict actual attributions made in response to the negative (i.e., failure) peer evaluation feedback. Conversely, we expected that children's enhancing, but not depressogenic attributional style would predict actual attributions made in response to the positive (i.e., success) peer evaluation feedback. For participants randomized to the neutral feedback condition we expected no linkage between CASQ-scores and cognitive reactions.

\section{Method}

Participants

Participants were 92 children (48 boys, 44 girls) enrolled in 5th and 6th grade classes from two public elementary schools in the Netherlands, who were predominantly from a middle-class SES background. The participants were predominantly Caucasian $(92.7 \%)$ and ranged in age from 10 to 13 years $(M=11.0, S D=.61)$. For the initial sample of 134 children, classroom teachers sent parent permission letters home with children. Of the 129 letters returned $(96.3 \%), 92$ parents $(71.3 \%)$ gave their consent for their children to participate in the study, and $37(28.7 \%)$ declined. We obtained verbal permission to perform the study from the principal of the school and each child's teacher. Children were informed that they could decide not to participate at any time.

\section{Procedure}

In the first of two sessions, approximately one week apart, participants were administered the Children Attributional Styles Questionnaire (Seligman et al., 1984), and the Children Depression Inventory (CDI, Kovacs, 1981) in their regular classrooms during school hours. Moreover, participants' social standing in their peer group was assessed via a commonly used procedure, which asks children to indicate whom among 
their classmates they like most and whom they like least (Coie, Dodge, \& Coppotelli, 1982; Newcomb, Bukowski, \& Pattee, 1993). A research assistant read the directions aloud and children were encouraged to ask for help if they had questions or encountered problems completing the questionnaires. The first session lasted approximately $45 \mathrm{~min}$. The second session was carried out in a quiet room on the school grounds. Participants were told that their class was selected to take part in an Internet computer-contest called 'Survivor'.

\section{Survivor contest}

Upon arrival, the participant was seated in front of a laptop computer equipped with a web-cam to have their photo taken. Participants were told that their picture would allow all the children participating to see what each of the other contestants looked like. Prior to the start of the contest (Time 1), participants completed a baseline mood measure. In an attempt to add both to the credibility and the attractiveness of the contest, an eyecatching logo of the American TV-show appeared on the computer display. The objective and rules of the contest were presented on screen.

Participants were informed that they would be competing with four same-sex players of comparable age (all of them were computerized confederates) from four different schools in the same area, and that all players would be evaluated by a jury consisting of 16 members, 8 boys and 8 girls. Specifically, participants were explained that each juror would give them a score between 0 and 100, with higher scores reflecting higher levels of perceived likeability.

After receiving this information, participants were directed through a series of screens in which they were asked to answer a series of questions that would give the members of the jury and the other children information about them. To minimize the possibility that children would respond to personal questions in a social desirable fashion, the importance of being honest and sincere was stressed. Moreover, it was explained that in all likelihood the jurors would differ markedly in their appreciation of other children's personal features (e.g., being lazy vs. being diligent). Finally, for most questions the "best" or "optimal" answer was unclear (e.g., "what is your favorite leisure activity"?).

Specifically, participants responded to questions about their favorite musical group, hobbies, preferred future occupation, things they liked and disliked about themselves, a number of character traits (e.g., perseverance), how they got along with other children, and their academic performance. Participants were informed on screen that their picture (previously taken by a web camera) along with the biographical information from their answers to the personal questions would be transmitted over the Internet and viewed by the jurors who would then give them a 'likeability' score ranging from 0 to 100 . Moreover, children were informed that the jurors would also indicate what they liked most and what they disliked most of each participant (e.g., "(s)he doesn't seem to be a nice person", "(s)he is witty", "(s)he is unattractive", "(s)he seems boring to hang out with").

Subsequent to answering all the biographical questions, participants were informed that pictures and descriptions of each of the other contestants would be presented one-at-a-time for review. To enhance credibility of the bogus contestants, actual selfdescriptions were taken from those of same-age children participating in another study. These participants gave their explicit consent to have this information viewed by other children, provided that the alleged self-description profiles would contain randomly combined personal information from at least three different children. 
Following the participant scrutinizing the last profile, a message appeared on the screen indicating that the computer would now for every child add the scores from the jurors to determine which contestant had received the highest total score and which contestant had received the lowest total score. After a $5 \mathrm{sec}$ waiting period, the names of those with the highest and the lowest score appeared in capital letters on the screen. In the success condition, the name of the actual participant was displayed as having obtained the highest total score; one randomly chosen alleged contestant's name appeared as having obtained the lowest total score. Conversely, in the failure condition the name of the actual participant was displayed as having obtained the lowest total score, while one alleged contestant's name appeared as having obtained the highest total score. In the control condition, the actual participant received neither the highest nor the lowest score.

Five seconds after receiving feedback (Time 2), participants responded to several probes that were designed to assess cognitive appraisals of the feedback outcome (see Measures). Next, the participant was accompanied to an adjacent room where a female research assistant then debriefed the child thoroughly.

\section{Debriefing}

Each child was thoroughly debriefed with the aim of removing any lingering effects of the false feedback while participating in the Survivor contest. During the debriefing, the child was informed that the jurors, the other contestants and the received feedback were entirely fictitious and that this deception was a necessary part of the procedure. At the conclusion of the debriefing, participants were urged to observe complete secrecy by not talking with their classmates about the Survivor contest until all the other children had finished participating. To increase adherence to this instruction, children were asked to sign a non-disclosure agreement and were then provided a choice of one of several possible small gifts for participating (e.g., a gift certificate worth about 3 dollars).

At the end of the debriefing, participants were encouraged to ask questions or voice their concerns. All children reported that they understood the purposes of the research, as well as the necessity of having been deceived. Most importantly, when asked, none of the participants made mention of any feelings of regret with regard to participation and none reported any objections to the procedure. The credibility of the deception manipulation was also assessed by asking each participant whether they had believed that they were competing against other children. With no exception, participants indicated that they had believed that the contest was genuine. Finally, all participants reported that prior to participating they had not talked with their classmates about the Survivor contest.

Measures

Children's Attributional Styles Questionnaire (CASQ; Seligman, Peterson, Kaslow, Tanenbaum, Alloy, \& Abramson, 1984)

The CASQ is a self-report inventory consisting of 48 items, 24 positive in nature and 24 negative in nature. Each item describes a hypothetical event (e.g., "you put a hard puzzle together"), and respondents are asked to imagine that the event happens to themselves. Respondents are presented with two possible causes for each event and asked to indicate which cause best reflects the way they would think if the event would 
actually happen to themselves. The two possible causes differ in one of three attributional dimensions examined (i.e., stable-unstable, internal-external, and globalspecific), and each dimension is tapped by 8 items. Scores on the two subscales (i.e., positive and negative composite) can range from 0 to 24. Several studies (e.g., Hilsman \& Garber, 1995) have reported moderate internal consistency for both the negative and the positive subscale. Scores on the CASQ were fairly consistent over a 6-month-period $(r=.66, p<.001$; Seligman et al., 1984), suggesting that attributional style among elementary school children is a relatively stable dispositional variable. The instrument has demonstrated good concurrent/criterion validity, in that children exhibiting higher levels of depressive symptoms are more likely than their peers to endorse global, stable, and internal explanations for negative evens, and more specific, unstable, and external explanations for positive events.

For the purposes of the present study, consistent with the hopelessness theory of depression (Abramson et al., 1989) and the recovery model of depression (Needles \& Abramson, 1990), analyses using the CASQ were limited to the attributional components of stability and globality for negative events, and the attributional components of stability and globality for positive events. The composite of negative stability and negative globality is referred to as depressogenic attributional style; the composite of positive stability and positive globality is referred to as enhancing attributional style.

In the present sample, Cronbach's alpha amounted to .53 for enhancing attributional style, and .59 for depressogenic attributional style, respectively. Total scores ranged from 1 to 11 on the subscale designed to assess enhancing style $(M=5.36, S D=2.10)$, and from 1 to 8 on the subscale designed to assess depressogenic style $(M=4.54$, $S D=1.95)$. Scores on both subscales did not differ as a function of age, gender, or their interaction.

\section{Children Depression Inventory (CDI; Kovacs, 1981)}

The CDI is a 27-item self-report measure designed to assess the social, behavioral, and affective symptoms of depression in children. Each item consists of three sentences that describe a symptom of depression in increasing degrees of severity. The respondent chooses the sentence that best describes him or her during the past week. Each item set is scored from 0 (symptom absent) to 2 (symptom is present always or most of the time). The CDI has adequate discriminant and convergent validity, test-retest reliability, and internal consistency (Saylor, Finch, Spirito, \& Bennett, 1984). Coefficient alpha in the present sample, using the Dutch version of the instrument (Braet \& Timbremont, 2002), was .82. Total scores ranged from 0 to 25 (median is 5). Scores were indicative of a nonclinical sample $(M=6.75, S D=5.03)$, and did not differ as a function of age, gender, or their interaction.

Social standing in the peer group

Participants completed a widely used nomination-based sociometric questionnaire, in which they identified the three classroom peers whom they liked most and the three classroom peers they disliked most (see Newcomb et al., 1993). The nominating pool comprised all children within a grade level, whereas nominations were made by participating children only. Within each grade level, the total number of nominations received by each child on the liked most and liked least questions were calculated and were transformed into standardized scores; i.e., LM and LL. Following Peery (1979), 
children's social standing score was then calculated by the formula LM-LL, yielding scores ranging from -5.40 to $3.07(S D=1.72)$. These scores did not differ as a function of gender, age, or their interaction.

Survivor contest state mood

Participants reported their state mood ("How do you feel right now?") on a 10-point Likert scale, ranging from 1 (extremely negative) to 10 (extremely positive). This measure was administered at the start of the game (Time 1) and five seconds postfeedback (Time 2).

Survivor contest cognitive reactions measure

A seven-item measure was developed to assess participants' cognitive appraisals/ evaluations of the feedback outcome. The first four items were designed to assess the dimension of stability by assessing participants' contest-related outcome expectancies for a subsequent round of Survivor. The specific items included: (a) "If I would participate again, but then against four other players, my total score would be..."; (b) "If I would participate again, but then with other jurors, my total score would be ..."; (c) "If I would participate again three weeks from now, my total score would be ..."; and (d) "If I would participate again, but then at home at my own computer, my total score would be ...". Participants rated their responses on the same 5-point Likert scale ( 1 = substantially higher, $2=$ somewhat higher, 3 = about the same, $4=$ somewhat lower, 5 = substantially lower).

The next three items were designed to assess the attributional dimension of globality by having children make judgments about the extent to which the peer evaluation outcome received during the Survivor contest matches their peer evaluations in other contexts. The specific items included: (a) "The outcome of Survivor is in line with my popularity in class"; (b) "The outcome of Survivor is in line with my popularity among peers that visit other schools"; and (c) "The outcome of Survivor is in line with my popularity among peers in general". Participants rated their responses on the same 5 -point Likert scale $(1=$ completely agree, $2=$ mostly agree, $3=$ somewhat agree, $4=$ mostly disagree, and $5=$ completely disagree). The mean scores for the stability and globality dimensions were calculated and then summed to yield one cognitive appraisal composite score, with higher scores reflecting more negative appraisals. Coefficient alpha for this composite was .68.

\section{Results}

Descriptive information and preliminary analyses

Means, standard deviations, and inter-correlations for all measures are presented in Tables 1 and 2. As displayed in Table 2, participants' scores on the positive and negative composite of the CASQ were not significantly associated. Conversely, children's scores on the CDI were significantly associated with their scores on both the positive and the negative composite of the CASQ. The data also showed a significant negative relationship between children's scores on the CDI and their peer nominated social standings ratings. None of these relationships was qualified by gender, age, or their interaction. 
Table 1 Means and standard deviations for all measures by condition

\begin{tabular}{|c|c|c|c|c|c|c|}
\hline & \multicolumn{2}{|c|}{ Success $(n=32)$} & \multicolumn{2}{|c|}{ Neutral $(n=28)$} & \multicolumn{2}{|c|}{ Failure $(n=32)$} \\
\hline & M & SD & M & SD & M & $\mathrm{SD}$ \\
\hline CASQ - Positive & 5.24 & 2.05 & 4.23 & 1.80 & 6.38 & 1.91 \\
\hline CASQ - Negative & 4.15 & 1.80 & 4.56 & 1.94 & 4.79 & 2.13 \\
\hline CDI & 7.27 & 6.10 & 5.80 & 4.48 & 7.13 & 4.43 \\
\hline Social Standing & .36 & 1.44 & -.79 & 2.33 & .50 & .94 \\
\hline Cognitive Appraisal - Stability & 3.05 & .39 & 2.40 & .67 & 2.21 & .52 \\
\hline Cognitive Appraisal - Globality & 2.58 & .71 & 2.39 & .64 & 3.04 & .72 \\
\hline Cognitive Appraisal - Total & 5.63 & .87 & 4.79 & 1.06 & 5.25 & .76 \\
\hline
\end{tabular}

Note: CASQ = Children's Attributional Styles Questionnaire; Children Depression Inventory

Table 2 Inter-correlations between CASQ, CDI, and social preference

\begin{tabular}{llll}
\hline & CASQ - Positive & CASQ - Negative & CDI \\
\hline CASQ - Negative & .10 & - & \\
CDI & $.40^{* *}$ & $.41^{* *}$ & - \\
Social Standing & -.05 & -.18 & $-.24 *$ \\
\hline
\end{tabular}

Note: CASQ = Children's Attributional Styles Questionnaire; Children Depression Inventory

$*=p<.05, * *=p<.01$

Equivalence of the experimental groups

Univariate ANOVA's comparing the three experimental conditions on baseline measures revealed no significant between-group differences, with the exception of the score on the enhancing subscale of the CASQ, $F(2,90)=9.29, p<.01, \eta^{2}=.17$ Posthoc multiple comparisons showed that children randomized to the failure feedback condition reported a weaker enhancing attributional style, relative to children randomized to receive either success $(p<.05)$ or neutral feedback $(p<.05)$.

Effects of peer feedback on state mood

Means and standard deviations for Time 1 and Time 2 state mood scores are presented in Table 2. A repeated measures ANOVA on these scores revealed a significant effect for Condition, $F(2,88)=29.31, p<.001, \eta^{2}=.40$. Subsequent simple effects analyses showed that children randomized to receive success feedback reported a significant improvement in state mood, $F(1,32)=55.56, p<.001, \eta^{2}=.64$; whereas children randomized to receive failure feedback reported a significant worsening in state mood, $F$ $(1,28)=19.72, p<.001, \eta^{2}=.41$. Children assigned to the neutral feedback condition reported no change in state mood $(p>.20)$. (Table 3$)$

Do children's actual attributions differ across feedback conditions?

Significant between-group effects were observed for both the stability $[F(2,90)=22.36$, $\left.p<.001, \eta^{2}=.33\right]$ and globality dimensions $\left[F(2,90)=7.56, p<.001, \eta^{2}=.14\right]$. Post-hoc multiple comparisons revealed that children randomized to the success feedback condition reported significantly lower outcome expectancies for a subsequent 
Table 3 Means and standard deviations for state mood at baseline (Time 1) and immediately postfeedback (Time 2) by condition

\begin{tabular}{|c|c|c|c|c|c|c|}
\hline & \multicolumn{2}{|c|}{ Success $(n=32)$} & \multicolumn{2}{|c|}{ Neutral $(n=28)$} & \multicolumn{2}{|c|}{ Failure $(n=32)$} \\
\hline & M & SD & M & SD & $\mathrm{M}$ & $\mathrm{SD}$ \\
\hline State Mood Time 1 & 7.94 & 1.37 & 8.04 & 1.06 & 8.04 & 1.70 \\
\hline State Mood Time 2 & 9.00 & 1.05 & 8.28 & 1.16 & 6.48 & 2.20 \\
\hline
\end{tabular}

round of Survivor (stability dimension), relative to children randomized to receive either neutral or failure feedback ( $p$ 's $<.001)$. Conversely, children randomized to the failure feedback condition scored significantly lower on the dimension of globality (i.e., extent to which their Survivor peer evaluation outcome is likely to match peer evaluations in other contexts), relative to children randomized to receive either neutral or success feedback $(p$ 's $<.01)$.

Is change in state mood predicted by attributional style or event-specific attributions?

We performed separate hierarchical regression analyses for the success and the failure condition. In these analyses, state mood score at Time 2 (post-contest) served as the dependent variable. For those randomized to the success condition, state mood score at Time 1 (pre-contest) was entered as a covariate in Step 1. In Step 2, CASQ enhancing attributional style score and actual attribution score for the positive Survivor feedback outcome were entered simultaneously. As recommended by Joiner (1994), we tested the assumption of homogeneity of covariance (Cohen \& Cohen, 1983) by also entering the two two-way interactions involving the covariate (e.g., state mood Time 1 by enhancing attributional style score) in Step 2. A similar analysis was performed for those randomized to the failure condition with the exception that CASQ depressogenic attributional style score and actual attribution score for the negative Survivor feedback outcome were entered in Step 2. Both analyses revealed no significant effects for either attributional style or actual event-specific attributions on changes in state mood. No violation of the assumption of homogeneity of covariance was observed in either case.

CASQ scores predicting children's cognitive appraisals of the feedback manipulation

In order to examine the predictive validity of children's attributional style for negative events as well as their attributional style for positive events, separate hierarchical regression analyses were performed for the success, failure, and the neutral feedback condition. In these analyses, event-specific attribution scores served as the dependent variable. In Step 1, baseline mood, age, gender, social standing score and CDI score were entered. The two CASQ subscale composite scores were entered in Step 2.

In response to the negative peer evaluation feedback, children displaying higher levels of depressive symptoms were significantly more likely to construe the rejection experience in a more negative fashion, $\beta=.46, R_{\text {change }}^{2}=.21, F_{\text {change }}=7.91, p<.01$. Above and beyond this effect for depression, children's scores on the CASQ negative (depressogenic) composite accounted for additional variance in endorsement ratings; $\beta=.41, R_{\text {change }}^{2}=.12, F_{\text {change }}=5.54, p<.03$. 
In response to the neutral peer evaluation feedback, none of the variables examined accounted for significant variance in these cognitive appraisal ratings. Finally, contrary to expectation, in response to the positive peer evaluation outcome, none of the variables examined accounted for significant variance in cognitive appraisal ratings.

\section{Discussion}

The present study sought to extend previous research on the psychometric properties of the CASQ. To our knowledge, this is the first study to investigate the linkages between children's CASQ scores and their cognitive appraisal of an actually experienced ecologically relevant (i.e., ego-involving) event in real time. Data on participants' changes in state mood revealed that our peer evaluation feedback manipulation was successful in eliciting differential affective reactions in the expected direction as a function of feedback valence. Moreover, our debriefing interviews indicated that children were involved in the Survivor contest and that none of the participants reported being aware that the feedback they received was bogus. Taken together, these data suggest that the Survivor feedback outcome was successful in achieving its major objective of providing a credible and salient event for the examination of the predictive validity of the CASQ.

Consistent with expectations, our findings revealed that children displaying a more depressogenic attributional style were more likely to evaluate their actual performance in a more negative fashion. Specifically, in response to Survivor failure feedback, above and beyond the effects of depression, the cognitive appraisals of children with higher depressogenic attributional style scores were more negative than those of their peers. Also consistent with expectations, children's scores on the CASQ were not associated with their cognitive appraisals of the neutral peer feedback outcome. However, contrary to expectations, children's enhancing attributional style as assessed by the CASQ did not predict actual attributions made in response to Survivor success feedback. Taken together, these findings provide partial support for the predictive validity of the instrument.

Interestingly, our findings showed that neither habitual attributional style nor actual event-specific attributions are linked to the magnitude of the momentary affective reactions to either the success or the failure feedback. Although these findings are at odds with research among adults providing evidence to suggest that the perceived cause(s) of success or failure experiences influence affective reactions to these outcomes (e.g., Weiner, Russell, \& Lerman, 1978, 1979; McFarland \& Ross, 1982), it should be noted that these studies employed a methodology in which participants were presented with hypothetical achievement outcomes along with a causal explanation for the outcome, and were then asked to indicate what their affective reactions to these situations would be. Hence, these findings do not speak to the role of attribution processes in influencing affective reactions in real life settings. Moreover, several studies that have examined the linkage between attributions and affective reactions in the context of an actual achievement outcome in real time, such as a midterm examination, also found that causal attributions are not important determinants of affective reactions among adults (e.g., McMillan \& Forsyth, 1983; Russell \& McAuley, 1986).

Several interesting findings emerged concerning the relationship between children's level of depression and their subsequent cognitive reactions to the peer evaluation feedback. Not surprisingly, children reporting higher levels of depression were more likely to construe the negative peer feedback outcome in a more pessimistic fashion. 
However, our data showed that children's cognitive appraisals of positive peer feedback were not affected by their level of depression. This latter observation is contrary to the well-documented association (which was also found in the present research) between higher levels of depression and a weaker enhancing attributional style for positive events, as assessed by the CASQ (see Gladstone \& Kaslow, 1995, for a review).

What might account for these discrepant findings for depression? One explanation is that the present peer success event differs from the types of positive scenarios included in the CASQ. Specifically, the success manipulation employed in the present study may have been more personally relevant (i.e., ego-involving), relative to the less personally relevant stimulus situations included in the CASQ (e.g., "you go to an amusement park and you have a good time") and hence elicit a different pattern of cognitive appraisals among children with elevated depression.

The difference in assessment methodology (i.e., non-current vs. online) is another possible explanation. According to the accessibility model of emotional self-report advanced by Robinson and Clore (2002), discrepancies often occur when people are asked to report on reactions to events they are currently experiencing, versus events they are not currently experiencing, because different factors contribute to self-reports under different reporting conditions. Specifically, online obtained reactions are largely governed by the appraisal of current situational conditions, which are episodic, contextual, and experiential in nature. Conversely, when reporting on reactions to not currently experienced emotion-eliciting events, people's self-reports are influenced by sources of non-experiential information, including situation-specific beliefs and personrelated beliefs. By extension, the vignette methodology may not capture individual differences as indexed by momentary assessments. In line with this formulation, Robinson and Johnson (1997) observed marked gender differences in adult participants' predictions of their negative emotional reactions to hypothetical vignettes, with women estimating that their emotions would be more intense than men's, whereas similar ratings obtained in an online condition did not vary by the gender of the participant.

The ecological validity of our peer manipulation procedure deserves further comment. We acknowledge that our laboratory manipulation is not identical to the peer rejection and peer praise experiences that children in this age range typically encounter in their daily lives. However, especially during the past decade, being evaluated while playing a game with unfamiliar peers has become widespread in television shows, and should by now probably be considered part of young adolescents' contemporary daily life.

Several limitations of the present study should be addressed in future work. First, because all significant results are based on children's self-report, we cannot rule out the possibility that our findings are partly due to shared method variance. Second, our findings are based on a community sample of children, rather than a clinical sample with a diagnosed mood disorder. Children's mean scores on the CDI suggest that depressive symptoms were modest in magnitude. It is therefore an empirical question to what extent our findings can be generalized to children who meet criteria for major depressive disorder. Third, it should be noted that cognitive vulnerability as defined by hopelessness theory of depression encompasses causal attributions, as well as negative inferences with regard to consequences and self-characteristics. Hence, future work examining the linkages between these other dimensions and event-specific inferences appears warranted. Finally, our investigation focused on children's cognitive reactions to only one important domain of negative emotion-eliciting events, namely peer rejection. While rejection has the benefit of being both a potent elicitor of negative 
affect as well as being ecologically sound, we should not assume that our findings can be generalized to other relevant domains such as academic failure, interpersonal conflict, and loss or separation.

\section{References}

Abela, J. R. Z. (2001). The hopelessness theory of depression: A test of the diathesis-stress and causal mediation components in third and seventh grade children. Journal of Abnormal Child Psychology, $29,241-254$.

Abramson, L. Y., Metalsky, G. L., \& Alloy, L. B. (1989). Hopelessness depression: A theory based subtype of depression. Psychological Review, 96, 358-372.

Abramson, L. Y., Seligman, S. E. P., \& Teasdale, J. D. (1978). Learned helplessness in humans: Critique and reformulation. Journal of Abnormal Psychology, 87, 49-74.

Beck, A. T. (1967). Depression: Clinical, experimental, and theoretical aspects. New York: Harper \& Row.

Braet, C., \& Timbremont, B. (2002). Children's depression inventory. Dutch version. Lisse: Swets \& Zeitlinger.

Cohen, J. (1977). Statistical power analysis for the behavioral sciences (rev. ed.). New York: Academic Press.

Cohen, J., \& Cohen, P. (1983). Applied multiple regression/correlation for the behavioural sciences (2nd ed.). Hillsdale, NJ: Erlbaum.

Coie, J. D. (1990). Toward a theory of peer rejection. In S. R. Asher \& J. D. Coie (Eds.), Peer rejection in childhood (pp. 365-401). New York: Cambridge University Press.

Coie, J. D., Dodge, K. A., \& Coppotelli, H. (1982). Dimensions and types of social status: A cross-age perspective. Developmental Psychology, 18, 557-580.

Cole, J. A., \& Turner, J. E. Jr. (1993). Models of cognitive mediation and moderation in child depression. Journal of Abnormal Psychology, 102, 271-281.

Dixon, J. F., \& Ahrens, A. H. (1992). Stress and attributional style as predictors of self-reported depression in children. Cognitive Therapy and Research, 16(6), 623-634.

Edelman, R. E., Ahrens, A. H., \& Haaga, D. A. F. (1994). Inferences about the self, attributions, and the overgeneralization as predictors of recovery from dysphoria. Cognitive Therapy and Research, 18, $551-566$.

Gladstone, T. R. G., \& Kaslow, N. J. (1995). Depression and attributions in children and adolescents: A meta-analytic review. Journal of Abnormal Child Psychology, 23, 597-606.

Gotlib, I. H., Lewinsohn, P. M., Seeley, J.R., Rohde, P., \& Redner, J. E. (1993). Negative cognitions and attributional style in depressed adolescents: An examination of stability and specificity. Journal of Abnormal Psychology, 102(4), 607-615.

Grusec, J. E., \& Lytton, H. (1988). Social development. New York: Springer-Verlag.

Hammen, C. L., Adrian, C., \& Hiroto, D. (1988). A longitudinal test of the attributional vulnerability model of depression in children at risk for depression. British Journal of Clinical Psychology, 27, 3746.

Hilsman, R., \& Garber, J. (1995). A test of the cognitive diathesis-stress model of depression in children: Academic stressors, attributional style, perceived competence, and control. Journal of Personality and Social Psychology, 69, 370-380.

Johnson, J. G., Crofton, A., \& Feinstein, S. B. (1996). Enhancing attributional style and positive life events predict increased hopefulness among depressed psychiatric inpatients. Motivation and Emotion, 20, 285-297.

Johnson, J. G., Han, Y. S., Douglas, C. J., Johannet, C. M., \& Russell, T. (1998). Attributions for positive life events predict recovery from depression among psychiatric inpatients: an investigation of the Needles and Abramson model of recovery from depression. Journal of Consulting and Clinical Psychology, 66, 369-376.

Joiner, T. E. Jr. (1994). Covariance of baseline symptom scores in prediction of future symptoms scores A methodological note. Cognitive Therapy and Research, 18(5), 497-504.

Joiner, T. E. Jr., \& Rudd, D. M. (1996). Toward a categorization of depression-related psychological constructs. Cognitive Therapy and Research, 20 (1), 51-68.

Joiner, T. E. Jr., \& Wagner, K. D. (1995). Attributional style and depression in children and adolescents: A meta-analytic review. Clinical Psychology Review, 15(8), 777-798. 
Kovacs, M. (1981). Rating scales to assess depression in school-aged children. Acta Paedo Psychiatra, 46, 305-315.

McFarland, C., \& Ross, M. (1982). Impact of causal attributions on affective reactions to success and failure. Journal of Personality and Social Psychology, 43, 937-946.

McMillan, J. H., \& Forsyth, D. R. (1983). Attribution-affect relationships following classroom performance. Contemporary Educational Psychology, 8, 109-118.

Monroe, S.M., \& Simons, A.D. (1991). Diathesis-stress theories in the context of life stress - Implications for the depressive disorders. Psychological Bulletin, 110, 406-425.

Needles, D. J., \& Abramson, L. Y. (1990). Positive life events, attributional style, and hopefulness: Testing a model of recovery from depression. Journal of Abnormal Psychology, 99, 156-165.

Newcomb, A. F., Bukowski, W. M., \& Pattee, L. (1993). Children's peer relations: A meta-analytic review of popular, rejected, neglected, controversial, and average sociometric Status. Psychological Bulletin, 113, 99-128.

Nolen-Hoeksema, S., Girgus, J. S., \& Seligman, M. E. P. (1986). Learned helplessness in children: A longitudinal study of depression, achievement, and explanatory style. Journal of Personality and Social Psychology, 51, 435-442.

Nolen-Hoeksema, S., Girgus, J. S., \& Seligman, M. E. P. (1992). Predictors and consequences of childhood depressive symptoms: A 5-year longitudinal study. Journal of Abnormal Psychology, 101, 405-422.

Panak, W. F., \& Garber, J. (1992). Role of aggression, rejection, and attributions in the prediction of depression in children. Development and Psychopathology, 4, 145-165.

Peery, J. (1979). Popular, amiable, isolated, rejected: A reconceptualization of sociometric status in preschool children. Child Development, 50, 1231-1234.

Robinson, M. D., \& Clore, G. L. (2002). Belief and feeling: Evidence for an accessibility model of emotional self-report. Psychological Bulletin, 128, 934-960.

Robinson, M. D., \& Johnson, J. T. (1997). Is it emotion or is it stress? Gender stereotypes and the perception of subjective experience. Sex Roles, 36, 235-258.

Russell, E., \& McAuley, E. (1986). Causal attributions, causal dimensions, and affective reactions to success and failure. Journal of Personality and Social Psychology, 50, 1174-1185.

Saylor, C. A., Finch, A. J., Spirito, A., \& Bennett, B. (1984). The Child Depression Inventory: A systematic evaluation of psychometric properties. Journal of Consulting and Clinical Psychology, 52, 955-967.

Seligman, M. E. P., Peterson, C., Kaslow, N. J., Tanenbaun, R. L., Alloy, L. B., \& Abramson, L. Y. (1984). Explanatory style and depressive symptoms among school children. Journal of Abnormal Psychology, 93, 235-238.

Sweeney, P. D., Anderson, K., \& Bailey, S. (1986). Attributional style in depression: A Meta-analytic review. Journal of Personality and Social Psychology, 50, 974-991.

Voeltz, Z. R., Haeffel, G. J., Joiner, T. Jr., \& Wagner, K. D. (2003). Reducing hopelessness: The interaction of enhancing and depressogenic attributional styles for positive and negative life events among youth psychiatric inpatients. Behaviour Research and Therapy, 41, 1183-1198.

Weiner, B., Russell, D., \& Lerman, D. (1978). Affective consequences of causal ascriptions. In J. H. Harvey, W. J. Ickes, \& R. F. Kidd (Eds.), New directions in attribution research (Vol. 2, pp. 59-90). Hillsdale, NJ: Erlbaum .

Weiner, B., Russell, D., \& Lermann, D. (1979). The cognition-emotion process in achievement-related contexts. Journal of Personality and Social Psychology, 37, 1211-1220. 